\title{
Conducting and Analyzing Empirical Studies in Search-Based Software Engineering
}

\author{
Lionel Briand \\ Simula Research Labs, Norway \\ briand@simula.no
}

\begin{abstract}
Search-Based Software Engineering (SBSE) has shown itself to be a promising and practical approach to address many long-standing software engineering problems (e.g., test case generation, automatic bug fixing, release planning). They must, however, be carefully evaluated through empirical studies, for example in terms of cost-effectiveness and scalability. Indeed, in most cases, there exist alternatives to solutions based on search, and a careful comparison is typically needed in order to better understand under which conditions each technique can be expected to perform best.

However, because search algorithms are randomized (e.g., metaheuristic search) and many contextual factors can affect their outcome, designing, running, and analyzing such empirical studies is fraught with issues and potential threats to validity. This tutorial aims at providing a number of introductory and fundamental principles to guide the design, execution, and analysis of empirical studies in SBSE. Though such principles will in many cases apply to contexts other than SBSE, the tutorial will target issues that are specific to that realm of research and use representative examples from its literature.
\end{abstract}

